\title{
New High Pressure Polymerization and Simultaneous Processing for Polyaminoimide from Aromatic Diamine and Bismaleimide
}

\author{
Kazuo Itoya, Yoshihiro Kumagai, Masami Kanamaru, \\ Hidetsugu Sawada, Masa-aki KaKimoto, Yoshio Imai ${ }^{\dagger}$, \\ and Osamu FukunagA* \\ Department of Organic and Polymeric Materials, and * Department of Inorganic Materials, \\ Tokyo Institute of Technology, Meguro-ku, Tokyo 152, Japan
}

(Received March 12, 1993)

\begin{abstract}
The simultaneous polymerization and processing under high pressure was carried out by using a piston-cylinder type hot pressing apparatus. Before the polymer synthesis, the Michael addition of aniline to $N$-phenylmaleimide was performed under high pressure as a model reaction, giving $N, N^{\prime}$-diphenylaspartimide. The Michael-type polyaddition of 4,4'-methylenedianiline to $4,4^{\prime}$-bismaleimidodiphenylmethane under $150-820 \mathrm{MPa}$ at $180-300^{\circ} \mathrm{C}$ for $20-40 \mathrm{~h}$ afforded the linear polyaminoimide having inherent viscosities in the range of $0.2-0.8 \mathrm{dlg}^{-1}$. When the polymerization was carried out at higher temperature, the closslinked polymer was produced. The polymerization under higher pressure required higher temperature. The closslinked polymer synthesized under high pressure was very hard resin with Vickers hardness of $330-360 \mathrm{MPa}$ and high modulus ( $>1.5 \mathrm{GPa}$ ), compared with the polymer obtained under ordinary pressure.

KEY WORDS High Pressure Polymerization / Polyaminoimide / MichaelType Polyaddition / Bismaleimide /
\end{abstract}

Polyaminoimide resins derived from aromatic bismaleimides and aromatic diamines are known as a class of high temperature thermosetting addition-type polyimides, and used widely as matrices for composite in electronics and aerospace industries. ${ }^{1-3}$ The advantage is that they contain reactive maleimide groups, which are thermally polymerizable without the elimination of volatile by-products that cause voids in highly closslinked polymeric materials.

In the field of synthetic polymer chemistry, the reactive hot pressing technique has been developed for many years. ${ }^{4,5}$ The concept of this technique is to simultaneously synthesize and mold intractable polymeric materials directly from solid monomers, and the technique was applied to the polymerization-

\footnotetext{
† To whom correspondence should be addressed.
}

processing of high temperature aromatic polymers. The application of high pressure to Michael addition reactions has been demonstrated to be extremely effective means due to both kinetic and thermodynamic backgrounds. ${ }^{6,7}$ We already reported the application of high pressure to the cycloaddition polymerization of $p$-cyanobenzonitrile $N$-oxide and found that the solid state polymerization at room temperature giving poly-1,2,4-oxaziazole was accelerated under high pressure. ${ }^{8}$ The present investigation was undertaken to explore high pressure polymerization and simultaneous processing for the polyaminoimide by the Michael-type polyaddition of $4,4^{\prime}$-bismaleimidodiphenylmethane (BMI) to $4,4^{\prime}$-methylenedianiline (MDI) without use of both solvent and catalyst [eq 1]. 


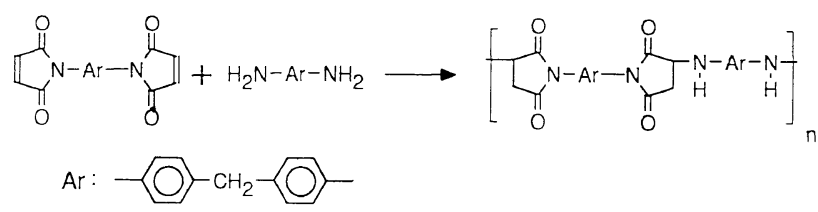

\section{EXPERIMENTAL}

\section{Apparatus}

The apparatus used for the high pressure reaction was the same as that described previously. $^{8}$

\section{Materials}

Commercial 4,4'-bismaleimidodiphenylmethane (BMI) and $N$-phenylmaleimide were recrystallized from a mixture of chloroform and methanol, and $o$-dichlorobenzene, respectively. 4,4'-Methylenedianiline (MDA), $m$ cresol and aniline were purified by distillation under reduced pressure.

\section{Model Reaction}

A $0.396 \mathrm{~g}(4.0 \mathrm{mmol})$ portion of $N$-phenylmaleimide and $0.409 \mathrm{~g}(4.4 \mathrm{mmol})$ aniline were placed into a Teflon capsule $(10 \mathrm{~mm}$ inside diameter) and then the capsule was loaded into the cylinder. The piston-cylinder was set at the furnace and the pressure was applied at room temperature to the monomer, followed by increasing temperature. The reaction was carried out at a different temperature between $20^{\circ} \mathrm{C}$ and $150^{\circ} \mathrm{C}$ for $3-70 \mathrm{~h}$ under a pressure of $0.1-500 \mathrm{MPa}$, giving $N, N^{\prime}$-diphenylaspartimide. The reaction temperature was recorded from the point where the temperature was reached to the prescribed one, which usually required around $1 \mathrm{~h}$. After the reaction, the temperature was lowered to room temperature, followed by releasing the pressure to atmospheric one. The product was isolated by washing with alcohol, and the resulting needle crystals were filtered and dried. $\mathrm{mp} 214^{\circ} \mathrm{C}$ (lit. ${ }^{3}$ $\mathrm{mp} 214-215^{\circ} \mathrm{C}$ ). The infrared (IR) spectrum $(\mathrm{KBr})$ exhibited characteristic absorptions at $3388 \mathrm{~cm}^{-1}(\mathrm{~N}-\mathrm{H})$ and $1708 \mathrm{~cm}^{-1}(\mathrm{C}=\mathrm{O})$.

\section{Preparation of Monomer Mixture}

A $3.86 \mathrm{~g}(19.5 \mathrm{mmol})$ portion of MDA was placed in a flask and stirred at $60^{\circ} \mathrm{C}$ under nirogen. Then to this $6.99 \mathrm{~g}(19.5 \mathrm{mmol})$ of powdered BMI was added and the mixture was stirred at that temperature for $20 \mathrm{~min}$, giving a homogeneous amber-colored mixture. The mixture was cooled to room temperature and used as the starting material for subsequent polymerizations. This had an inherent viscosity of $0.05 \mathrm{dl} \mathrm{g}^{-1}$, measured at a concentration of $0.5 \mathrm{~g} \mathrm{dl}^{-1}$ in methanesulfonic acid at $30^{\circ} \mathrm{C}$.

\section{Solution Polymerization}

The solution polymerization of BMI with MDA was conducted in $m$-cresol accoding to the method of Crivello. ${ }^{3}$ The inherent viscosity of the polymer obtained was $0.75 \mathrm{dlg}^{-1}$ in methanesulfonic acid.

\section{High Pressure Polymerization}

A Teflon capsule $(10 \mathrm{~mm}$ inside diameter $)$ was used for the polymerization of the monomer mixture $(0.5 \mathrm{~g})$. The polymerization was carried out at a different temperature between $150^{\circ} \mathrm{C}$ and $340^{\circ} \mathrm{C}$ for $5-20 \mathrm{~h}$ under a pressure of $0.1-800 \mathrm{MPa}$, giving polyaminoimide. After the reaction, the polymer in the form of a flat cylindrical pellet ( $10 \mathrm{~mm}$ diameter and $2-3 \mathrm{~mm}$ thick) was removed from the Teflon capsule.

\section{Measurement}

IR spectra were recorded on a JASCO FT/IR-5000 spectrophotometer. ${ }^{1} \mathrm{H}$ and ${ }^{13} \mathrm{C}$ NMR spectra were recorded on a JEOL JMN-FX90Q spectrometer. Differential scanning calorimetry (DSC) and thermogravimetry (TG) were performed with Shimadzu thermal analyzers DSC-41 and TGA-40, respectively. 
Dynamic mechanical measurements (DMA) were performed with a Toyoseiki Rheolograph Solid with specimen $(0.6 \mathrm{~cm}$ gauge length, $0.5 \mathrm{~cm}$ wide, and $300-500 \mu \mathrm{m}$ thick) at $10 \mathrm{~Hz}$ frequency at a heating rate of $3^{\circ} \mathrm{Cmin}^{-1}$. Density of the polymers was measured by a conventional Archimedes displacement method in water at $25^{\circ} \mathrm{C}$. Vickers hardness measurements were carried out at room temperature using a Matsuzawa Seiki MHT2 microhardness tester equipped with a diamond square pyramid, where a load of $500 \mathrm{~g}$ was applied for $15 \mathrm{~s}$.

\section{RESULTS AND DISCUSSION}

\section{Model Reaction}

$N, N^{\prime}$-Diphenylaspartimide (DMI) has already been prepared by the Michael addition of aniline to $N$-phenylmaleimide almost quantitatively. ${ }^{3}$ High pressure is also known to accelerate Michael reactions, however, there has been no report on the synthesis of DMI under high pressure, which was a model for the Michael-type polyaddition reaction [eq 2].<smiles>Cc1ccc(N2C(=O)C=C(Nc3ccccc3)C2=O)cc1</smiles>

The high pressure reaction was carried out in the absence of both solvent and catalyst by using a piston-cylinder type hot-pressing apparatus. Figure 1 shows the effect of applied pressure and reaction temperature on the yield of DMI formed by the reaction for $3 \mathrm{~h}$. DMI was not obtained under ordinary pressure at $20^{\circ} \mathrm{C}$, but was obtained when high pressure was applied. As the reaction temperature increased, the yield of DMI increased under both ordinary pressure and high pressure conditions. Figure 2 shows the effect of pressure on the yield of DMI by the reaction at $90^{\circ} \mathrm{C}$ for $3 \mathrm{~h}$. The yield increased with increasing pressure up to $300 \mathrm{MPa}$, however, the pressure higher than that tended to decrease the yield. These results

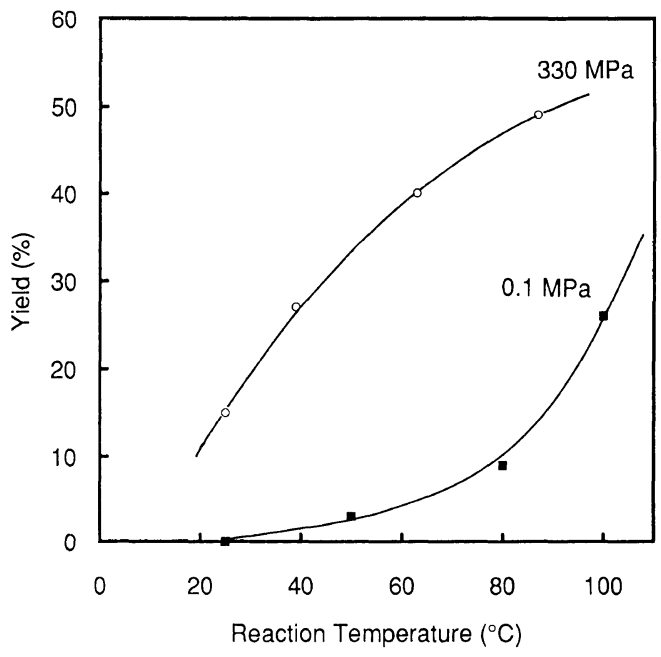

Figure 1. Effects of applied pressure and reaction temperature on the synthesis of $N, N^{\prime}$-diphenylaspartimide by the reaction of $N$-phenylmaleimide and aniline for $3 \mathrm{~h}$.

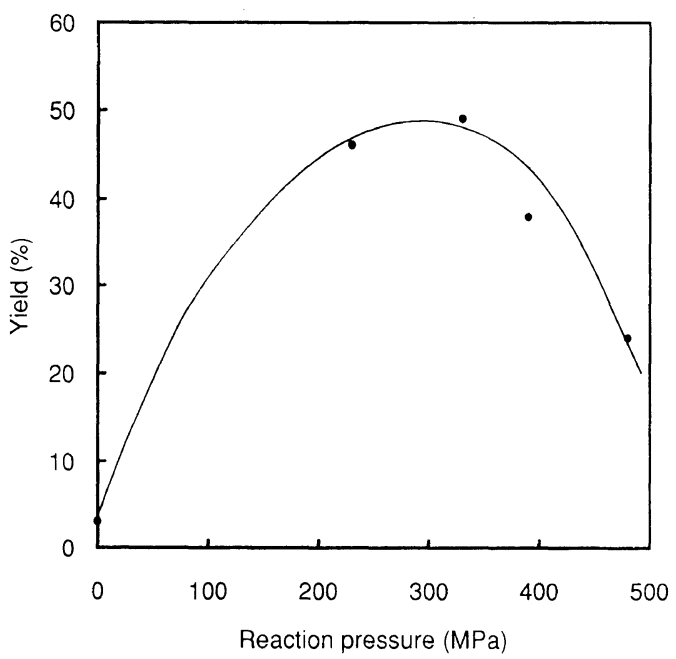

Figure 2. Effect of applied pressure on the synthesis of $N, N^{\prime}$-diphenylaspartimide by the reaction of $N$-phenylmaleimide and aniline at $90^{\circ} \mathrm{C}$ for $3 \mathrm{~h}$.

indicated that the pressure actually accelerated the Michael addition, whereas very high pressure around $500 \mathrm{MPa}$ had little effect on the reaction. This is probably associated with the melting points $(\mathrm{mp})$ of the two reactants, aniline and $N$-phenylmaleimide, which are $-6^{\circ} \mathrm{C}$ and $87^{\circ} \mathrm{C}$, respectively. Usually the 
reaction proceeded in a liquid state, because aniline acted as a solvent. It is known that the mp's of compounds increase with increasing applied pressure. When very high pressure was applied, the reaction mixture might solidify, giving a solid-solid phase which retarded the reaction. Thus, the Michael addition without use of solvent and catalyst was accelerated or proceeded smoothly under high pressure in a liquid state.

\section{Preparation of Monomer Mixture and Ordinary} Pressure Polymerization

Following the model reaction, the Michaeltype polyaddition of MDA to BMI was tested under high pressure. High-molecular-weight polymer could not be obtained by the high pressure polymerization of the mixture of two solid monomers, MDI and BMI, due to the fact that the contact and diffusion of two monomers were highly restricted under high pressure. For this reason, the monomers were mixed in advance in the molten state, and the homogeneous monomer mixture with an inherent viscosity of $0.05 \mathrm{dl} \mathrm{g}^{-1}$ was prepared and used for further polymerizations. Under ordinary pressure, this monomer mixture could be polymerized at a temperature of 140 $180^{\circ} \mathrm{C}$, giving linear polyaminoimide having inherent viscosity values between $0.2-0.8$ $\mathrm{dl} \mathrm{g}^{-1}$ in quantitative yield. The glass transition temperature $\left(T_{\mathrm{g}}\right)$ of the polymer synthesized under high pressure was $202^{\circ} \mathrm{C}$, determined by DSC at a heating rate of $10^{\circ} \mathrm{C} \mathrm{min}^{-1}$ in nitrogen, which was in good agreement with the $T_{\mathrm{g}}$ value of $211^{\circ} \mathrm{C}$ reported by Crivello. ${ }^{3}$

When the polymerization was carried out at $180{ }^{\circ} \mathrm{C}$ or a higher temperature, closslinked polyaminoimide was obtained. This closslinking reaction mechanism can be explained by the radical reaction of double bond of terminal maleimide moiety [eq 3 ] and the Michael addition of amino group in the polymer chain to the terminal maleimide group [eq 4].

The closslinked polymers formed was amorphous, very hard resin (Vickers hardness,
$280 \mathrm{MPa}$ ), and the initial decomposition temperature was $280^{\circ} \mathrm{C}$ in nitrogen.

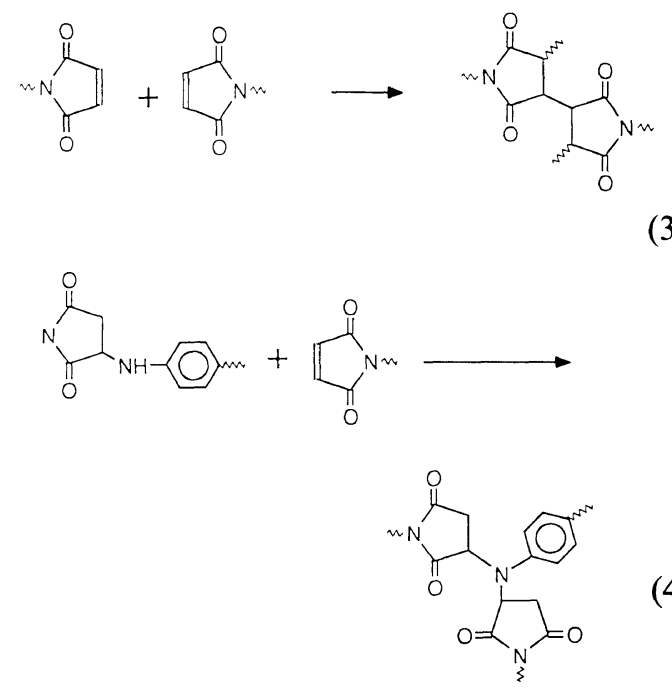

High Pressure Synthesis of Linear Polyamioimide

The results of the model reaction was most encouraging for the high pressure polymerization giving polyaminoimide. The high pressure polymerizations of the momomer mixture containing BMI and MDI were carried out under $150-820 \mathrm{MPa}$ at $180-320^{\circ} \mathrm{C}$ for $20-40 \mathrm{~h}$ without use of both solvent and catalyst, in order to find the boundary conditions (applied pressure and reaction temperature) to effect the polymerization. Figure 3 shows the results of the polymerization under high pressure for $20 \mathrm{~h}$. When a certain temperature was reached under a constant pressure, the monomer mixture polymerized to form transparent, brown-colored, brittle, and hard linear polyaminoimide resin. Since this boundary temperature probably corresponds to the softening point of the monomer mixture, the temperature to cause polymerization increased with increasing the applied pressure.

Figure 4 exhibits the effects of reaction temperature and applied pressure on inherent viscosity of the linear polyaminoimide obtained by the polymerization of the monomer mixture 


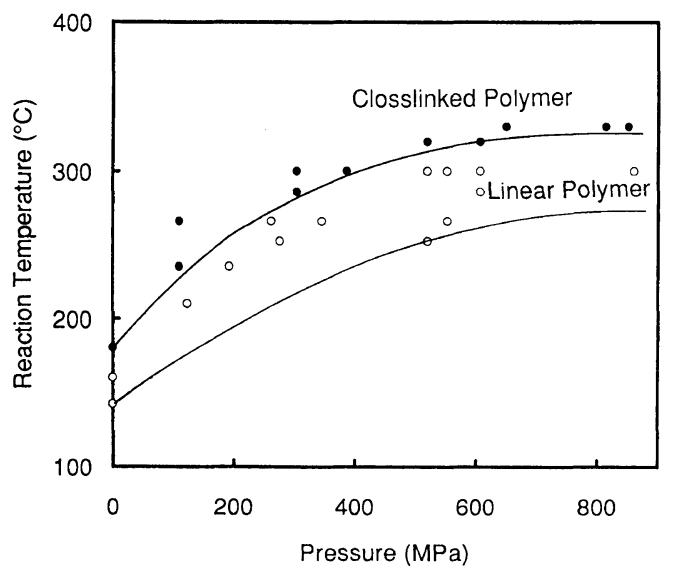

Figure 3. Effects of reaction temperature and applied pressure on the polymerization for $20 \mathrm{~h}$ giving polyaminoimide.

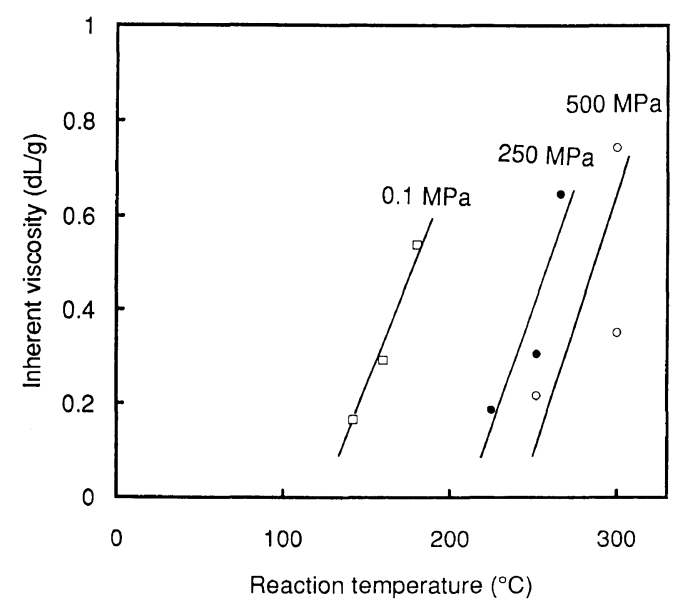

Figure 4. Effects of reaction temperature and applied pressure on inherent viscosity of polyaminoimide formed by the polymerization for $20 \mathrm{~h}$.

for $20 \mathrm{~h}$. The inherent viscosity of the polymer increased monotonically with increasing the reaction temperature under constant pressure, whereas it shifted to lower value with increasing the applied pressure. These results indicated that the polymerization under higher pressure required higher temperature to afford high molecular weight polymer.

The structure of the resulting polyaminoimide by high pressure polymerization was confirmed by comparing their IR and ${ }^{1} \mathrm{H}$ NMR

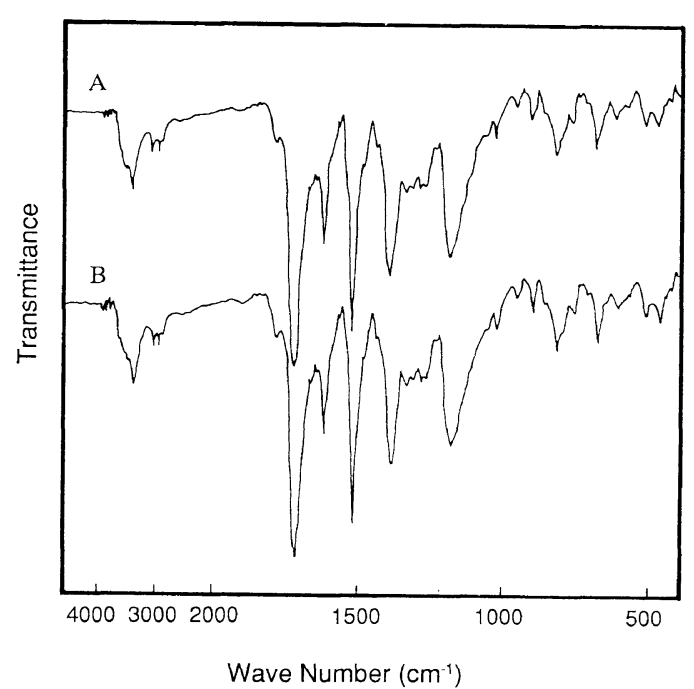

Figure 5. IR spectra of polyaminoimide: (A) the polymer formed by the solution polymerization; (B) the polymer formed by the high pressure polymerization.

spectra with those of the authentic polyaminoimide obtained by the solution polymerization according to the previously reported procedure. ${ }^{3}$ Figures 5 and 6 show the IR and 1H NMR spectra, respectively, of both high pressure polymerized and solution polymerized polyaminoimides. The IR and ${ }^{1} \mathrm{H}$ NMR spectra of both polyaminoimides agreed very closely with each other. In addition the TG curve of the polymer synthesized under high pressure was very similar in decomposition pattern with that of the solution polymerized polymer (Figure 7). These results clearly indicated that the high pressure polymerized polymer was the same as the polymer obtained by the solution method.

\section{High Pressure Synthesis and Properties of Closslinked Polyamioimide}

The closslinked polyaminoimide resin was usually obtained by the polymerization of the momoner mixture under $150-820 \mathrm{MPa}$ at $230-320^{\circ} \mathrm{C}$ for $20-40 \mathrm{~h}$. Figure 3 shows again the boundary conditions (applied pressure and reaction temperature) for the closslinked polymer formation. As the applied pressure 

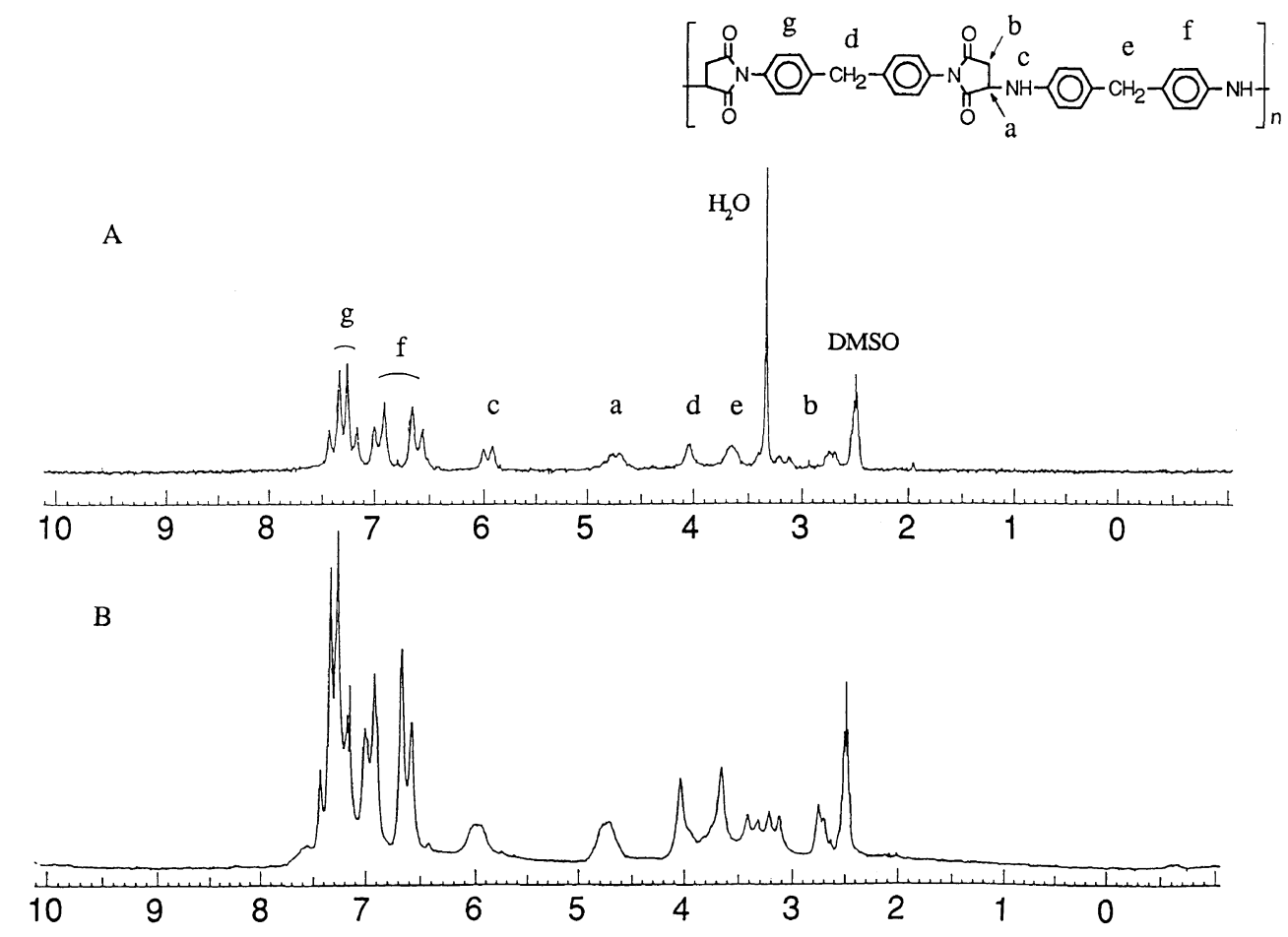

Figure 6. ${ }^{1} \mathrm{H}$ NMR spectra of polyaminoimide: (A) the polymer formed by the solution polymerization; (B) the polymer formed by the high pressure polymerization.

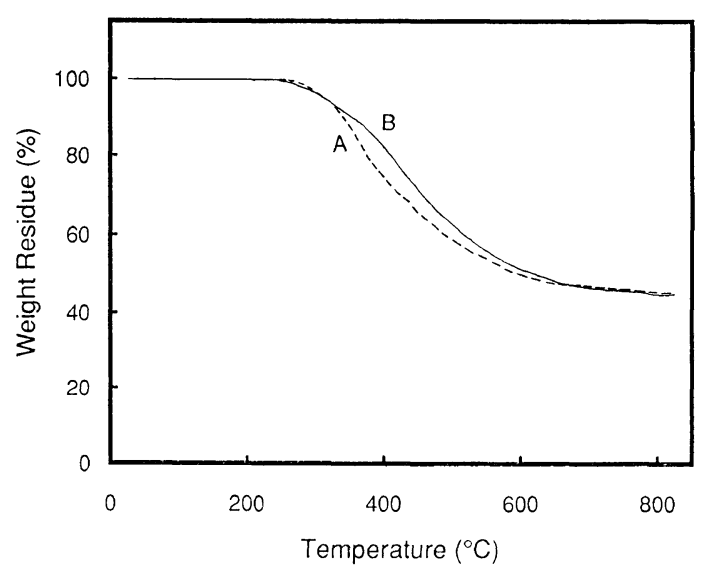

Figure 7. TG curves of polyamideimide at a heating rate of $10^{\circ} \mathrm{C} \mathrm{min}^{-1}$ in nitrogen: (A) the polymer formed by the solution polymerization; (B) the polymer formed by the high pressure polymerization.

increased, higher reaction temperature was required to obtain the closslinked polymer. The results suggested that this closslinking reaction could occur in a rubbery state of the polymer, because the boundary temperature probably corresponds to glass transition temperature of the linear polymer. The closslinked polymer obtained was transparent, amber-colored, and very hard polyaminoimide resin, and the decomposition temperature was $280^{\circ} \mathrm{C}$ in nitrogen, which was almost the same as that of the linear polyaminoimide.

Figure 8 exhibits pressure dependence of density of the polyamideimide. Although no marked difference in density of the linear polymer and the closslinked polymer was observed, the density of the polymer increased to some extent with increasing the applied pressure.

The dynamic mechanical studies are capable of detecting subtle difference in structure of the polymer that results from difference in polymerization conditions. Curve $\mathrm{A}$ in Figure 


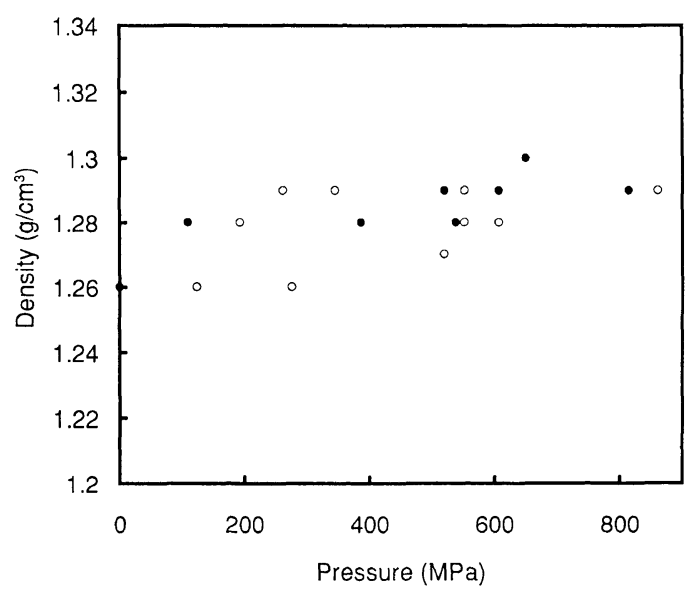

Figure 8. Pressure dependence of density of polyamideimide: $(\bigcirc)$ linear polymer; $(\bigcirc)$ closslinked polymer.

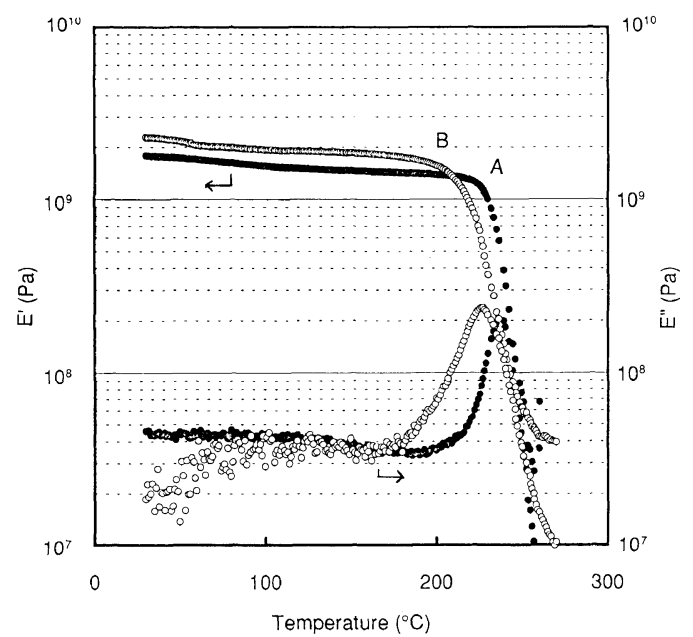

Figure 9. Temperature dependence curves of dynamic storge modulus $\left(E^{\prime}\right)$ and dynamic loss modulus $\left(E^{\prime \prime}\right)$ for polyaminoimide: (A) the polymer formed at $210^{\circ} \mathrm{C}$ under $0.1 \mathrm{MPa}$ for $20 \mathrm{~h}$; (B) the polymer formed at $300^{\circ} \mathrm{C}$ under $300 \mathrm{MPa}$ for $20 \mathrm{~h}$.

9 shows the dynamic mechanical properties of the polyaminoimide formed by the polymerization under ordinary pressure at $210^{\circ} \mathrm{C}$ for $20 \mathrm{~h}$. The dynamic loss modulus $\left(E^{\prime \prime}\right)$ curve of the polymer exhibited a rather narrow peak for $T_{\mathrm{g}}$ around $235^{\circ} \mathrm{C}$ and the dynamic storage modulus $\left(E^{\prime}\right)$ of polymer showed only a slight decrease up to $220^{\circ} \mathrm{C}$, suggesting that high molecular-weight lightly closslinked polymer

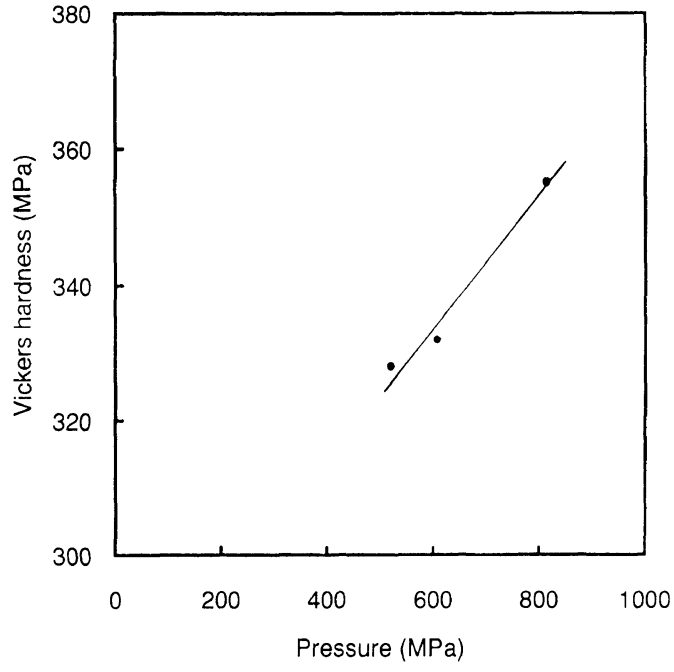

Figure 10. Effects of applied pressure on Vickers hardness of polyaminoimide formed by the polymerization at $320^{\circ} \mathrm{C}$ for $20 \mathrm{~h}$.

was obtained. Curve B in Figure 9 shows the $E^{\prime}$ value of the polymer formed at $300^{\circ} \mathrm{C}$ under $300 \mathrm{MPa}$ for $20 \mathrm{~h}$. As compared with curve A, the high pressure polymerized polymer had lower $T_{\mathrm{g}}$ of $225^{\circ} \mathrm{C}$ and higher storage modulus around $2.0 \mathrm{GPa}$ below $T_{\mathrm{g}}$, suggesting the formation of low-molecular-weight highly closslinked polymer.

More remarkable was the hardness value of the closslinked polymer formed under high pressure; the polymer had higher Vickers hardness $(>300 \mathrm{MPa})$ compared with the ordinary pressure processed polymer. Figure 10 shows the effect of applied pressure on Vickers Hardness of the polyaminoimide synthesized at $320^{\circ} \mathrm{C}$ for $20 \mathrm{~h}$, indicating that the hardness value increased with increasing the applied pressure. These results suggested that applied pressure made the polymers dense packing, giving compact resins with high modulus, high density, and high Vickers hardness values.

\section{CONCLUSIONS}

The Michael addition of aniline and 
$\mathrm{N}$-phenylmaleimide was first carried out as a model reaction, giving $N, N^{\prime}$-diphenylaspartimide. This reaction was accelerated under high pressure. The Michael-type polyaddition of MDA to BMI under $150-820 \mathrm{MPa}$ at $180-320^{\circ} \mathrm{C}$ for $20-40 \mathrm{~h}$ afforded the linear polyaminoimide having inherent viscosities in the range of $0.2-0.8 \mathrm{dl} \mathrm{g}^{-1}$. The polymerization under higher pressure required higher temperature. When the polymerization was carried out at higher temperature $\left(230-320^{\circ} \mathrm{C}\right)$ under $150-820 \mathrm{MPa}$, the crosslinked polymer was produced. As the applied pressure increased, higher reaction temperature was requiered to obtain the closslinked polymer. These results suggested that the polymerization became diffusion-limited under high pressure and hence the reaction rate decreased. The closslinked polymer synthesized under high pressure was found to be very hard resin with Vickers hardness of $330-360 \mathrm{MPa}$ and high modulus $(>1.5 \mathrm{GPa})$, compared with the polymer obtained under ordinary pressure. The high-pressure polymerized closslinked polyaminoimide could find new applications as high temperature thermosetting polyimide molding.

Acknowledgments. The authors gratefully acknowledge the support for this work by a Grant-in-Aid for Scientific Research on Priority Areas, New Functionality Materials, Design, Preparation, and Control (No. 2205042) from the Ministry of Education, Science, and Culture of Japan.

\section{REFERENCES}

1. P. E. Cassidy, "Thermally Stable Polymers," Dekker, New York, 1980.

2. D. Wilson, H. D. Stenzenberger, and P. M. Hergenrother, "Polyimides," Blackie, New York, 1990.

3. J. V. Crivello, J. Polym. Sci., Polym. Chem. Ed., 11, 1185 (1973).

4. P. E. D. Morgan and H. Scott, J. Polym. Sci., B, 7, 437 (1969).

5. P. E. D. Morgan and H. Scott, J. Appl. Polym. Sci., 16, 2029 (1972).

6. K. Matsumoto, A. Sera, and T. Uchida, Synthesis, 1 (1985).

7. K. Matsumoto and A. Sera, Synthesis, 999 (1985).

8. K. Itoya, M. Kakimoto, Y. Imai, and O. Fukunaga, Polym. J., 24, 979 (1992). 